\title{
Nonnatural Phospholipids: Probing Nature's Modular Platform
}

\author{
Andreas Zumbuehl*
}

\begin{abstract}
Life as we know it would not be possible without a cellular membrane, without a separation between two compartments. This plasma membrane, however, remains a difficult object to study directly. The synthesis of nonnatural phospholipids thus provides valuable probes for experiments at the membrane-level and integrates organic chemistry with basic biophysics and biology. A few of the concepts are highlighted in this brief overview.
\end{abstract}

Keywords: Biophysics · Membrane probes · Nonnatural phospholipids

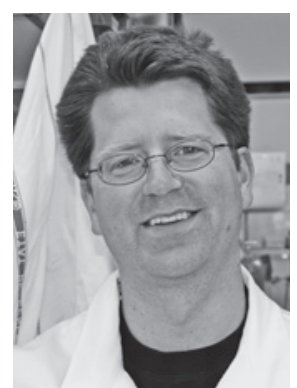

Andreas Zumbuehl was born in Interlaken (Switzerland) in 1974. He graduated from ETH Zürich in 1999 and also received his $\mathrm{PhD}$ from ETH in 2004 working under the guidance of Professor Erick M. Carreira. He then spent his postdoctoral years with Professor Robert Langer at the Massachusetts Institute of Technology in Cambridge (USA) and Professor Joachim Seelig at the Biozentrum Basel. In 2008 he became Maître Assistant at the University of Geneva where he started his independent research on the synthesis and applications of nonnatural phospholipids.

\section{Introduction}

The biological membrane is a complex barrier at the very heart (and beginning) of life. The membrane's major components are proteins and lipids in a mixture adapted to the needs of the current local environment. Whereas the importance of proteins has been recognized for a long time, lipids were regarded as a rather dull structural necessity of a cell's membrane. The best proof of this statement is the lack of a clear definition for one of Earth's oldest class of molecules: Lipids still are merely described by their physical properties, being virtually insoluble in water and readily soluble in organic solvents.

Only in recent years has the full significance of our lipidome, the thousands of structurally different lipids in our body, been appreciated and is currently been mapped by an international lipidomics initiative. ${ }^{[1]}$ This also opens the field for synthetic chemists (such as our group at the University of Geneva) trying to create variants of natural lipids and exploring their properties. Phospholipids can be synthesized with nonnatural tails, interface regions, and headgroups and these structural motifs will be briefly touched upon in this overview article (Fig. 1).

\section{Medicinal Applications}

One of the first interests in the field was motivated by creating serum-stable, phospholipase $\mathrm{A}_{2}$ resistant lipids and to explore their anti-cancer and later anti-HIV potential. The field was pioneered by de Haas and van Deenen in the early 1970s, who reported on the synthesis of several phospholipid analogues. ${ }^{[2]}$ The exchange of the

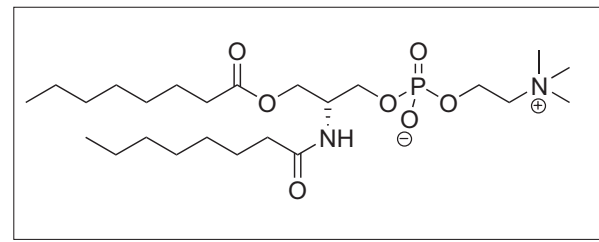

Fig. 2. Nonnatural phospholipid bearing an amide bond at the sn-2 position. This modification renders the molecule inert towards the cleavage by the enzyme phospholipase $\mathrm{A}_{2}$.

$s n-2$ ester with an amide led to inhibition of phospholipase $\mathrm{A}_{2}$ normally cleaving the lipid at this position (Fig. 2).

Another medicinal application of phospholipids is the liposomal formulation of drugs. The major problem remains the relative instability of the vesicles in vivo and a great deal of work therefore went into the development of polymerizable phospholipids that would yield stable polymeric nanoparticles (Fig. 3).[3] Both the groups of Chapman and Ringsdorf developed a series of diacetylene-modified phospholipids. ${ }^{[4]}$ These phospholipids have significantly lower $\mathrm{T}_{\mathrm{m}}$ compared to the natural phospholipids with no true collapse point or phase change observed in either calorimetric measurements or pressure-area
${ }^{\star}$ Correspondence: Dr. A. Zumbuehl Department of Organic Chemistry University of Geneva

30, quai Ernest Ansermet

$\mathrm{CH}-1211$ Genève 4

Tel.: + 41223796719

Fax: + 41223793215

E-mail: andreas.zumbuehı @unige.ch

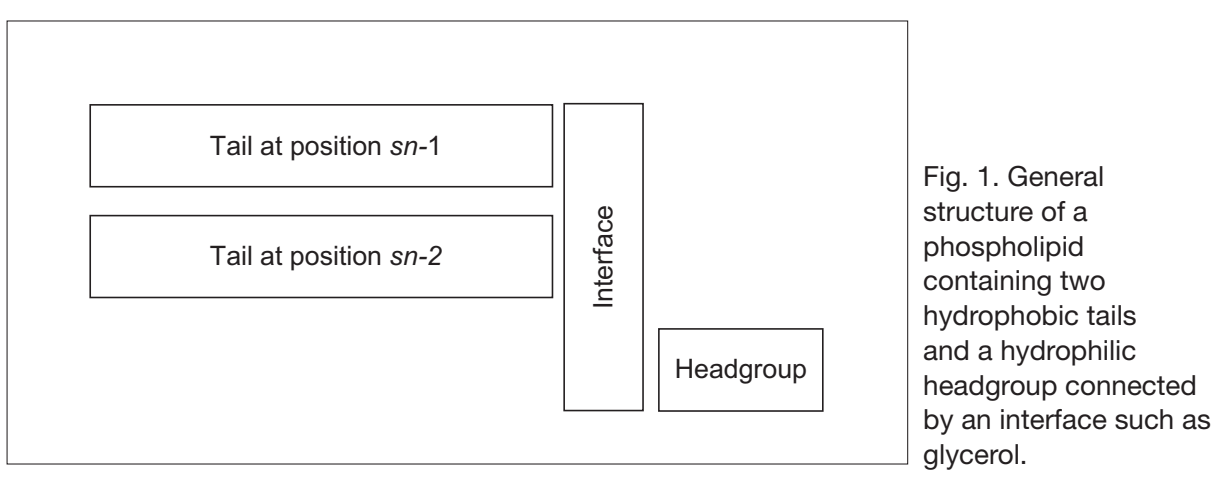




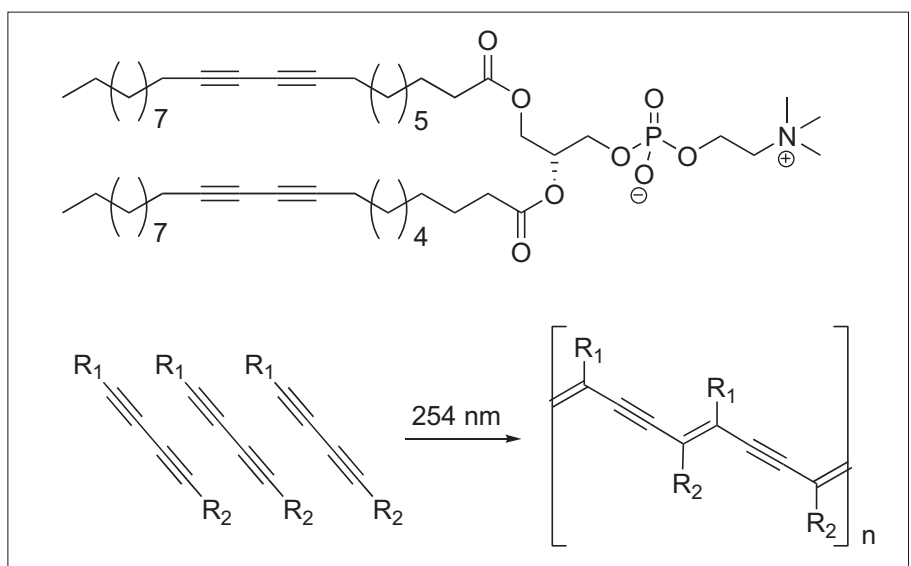

Fig. 3. Diacetylene phospholipids leading to two-dimensional polymerized vesicles or membranes.

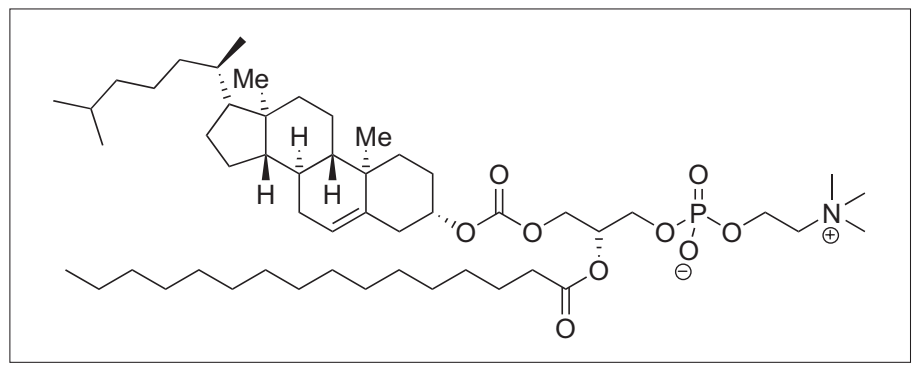

Fig. 4. A sterol-modified glycerophospholipid (SML), a hybrid between a cholesterol and a phospholipid.

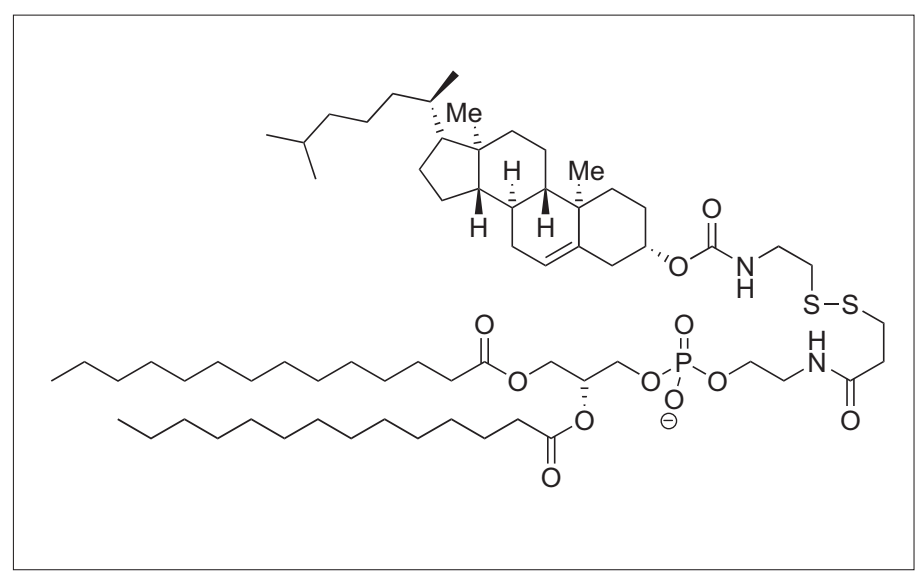

Fig. 5. Regen's nearest-neighbor recognition technique: A snapshot of the membrane structure is taken after oxidation of mixed free thiol reagents.

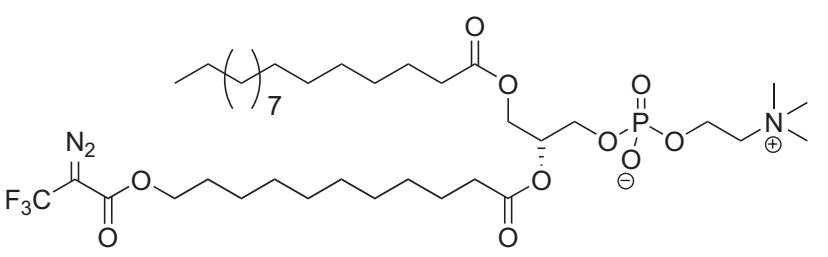

Fig. 6. Photoactive phospholipids containing $\omega$-diazo trifluoropropionyloxy groups. isotherms. The diacetylene moieties were less reactive compared to free diacetylenic fatty acids but nonetheless significant polymerization of vesicles could be induced at $254 \mathrm{~nm}$ at temperatures below the $\mathrm{T}_{\mathrm{m}}{ }^{[5]}$ Such polymers could have enormous potential as medicinal materials as they are essentially mimicking the natural plasma membrane. Indeed, polymers made of a truncated phosphocholine headgroup, 2-methacryloyloxyethyl phosphorylcholine (MPC), show favorable biocompatibility properties. ${ }^{[6]}$

Back to the problem of instable vesicles, Huang and Szoka Jr. recently presented another solution: They noted that the cholesterol formulated into liposomes would rapidly exchange into natural membranes in vivo leaving behind a destabilized vesicle. ${ }^{[7]}$ They therefore synthesized a sterol-modified glycerophospholipid (SML), essentially a hybrid between a cholesterol and a phospholipid (Fig. 4).[7] The SMLs retained the membrane-condensing properties of free cholesterol without the negative effect of sterol-exchange. Using an in vivo tumor model, a doxorubicin-loaded SML vesicle formulation was compared to the FDAapproved liposomal formulation (Doxil) and no significant difference between the two formulations was found. ${ }^{[7]}$ The new SML vesicle formulation might be easier to manufacture, as less components are needed for its preparation.

\section{Biophysical Studies}

The study of biophysical properties of the plasma membrane was another major driving force for the synthesis of nonnatural phospholipids. A classical example is the nearest-neighbor recognition developed by the group of Regen. ${ }^{[8]}$ They were able, for example, to show that cholesterol would favorably interact with phospholipids containing long alkyl chains (vs. shorter chains) and that it is probably not the phospholipid headgroup driving this interaction (Fig. 5). ${ }^{[8]}$

Lipid-lipid interaction studies were pioneered amongst others by the group of Khorana. Using carbene-functionalized phospholipids the group was for example interested in mapping the position of phospholipids in the membrane (Fig. 6). The experiments showed that the glycerol backbone must be tilted away from the membrane plane, because the chain at $s n-2$ would tail the chain at $s n-1$ by two to four atoms. ${ }^{[9]}$ Deuterated and ${ }^{31} \mathrm{P}$-labeled phospholipids synthesized by the group of Seelig showed that indeed the glycerol moiety is perpendicular to the membrane plane. In addition, it was shown that the phospho- choline headgroup and the first atoms of the $s n-2$ chain extend almost parallel to the membrane plane (Fig. 7). ${ }^{10]}$

Membrane proteins are influenced by their neighboring lipids. It is thus only natural to use nonnatural phospholipids as tools to study membrane protein-lipid interactions. The group of Brunner devised various phospholipid probes for the general labeling of the apolar core of membranes (Fig. 8). ${ }^{[11]}$ An aziridine probe can be activated by UV-light and will react with whatever protein segment would be nearest to

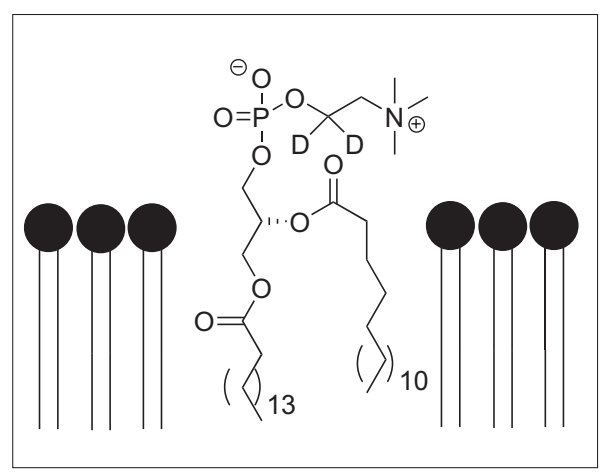

Fig. 7. Deuterated phospholipid probe for solid state NMR proving that the sn-2 ester is first extending parallel to the membrane plane to make an abrupt $90^{\circ}$ turn at $\mathrm{C}(2)$. The phosphocholine headgroup is also extending almost parallel to the membrane. 


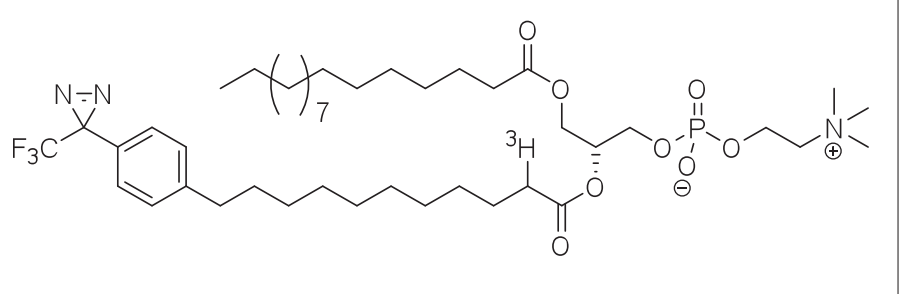

Fig. 8. Aziridine-labeled phospholipid as UV-active probe of the hydrophobic depths of a membrane.

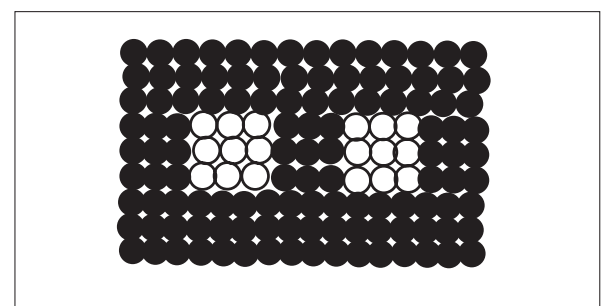

Fig. 10. Viewed from the top: Schematic drawing of a multi-well plate made of crosslinked solid-supported phospholipids (filled circles) providing the boundary for free floating 'natural' lipid bilayers (clear circles).

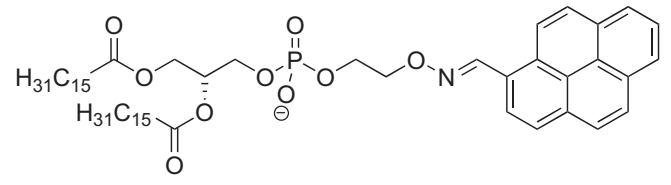

Fig. 9. Pyrene-functionalized phospholipid with an oxime linker.

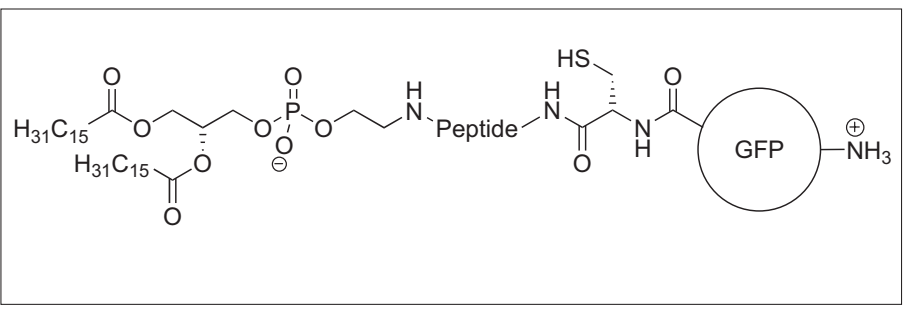

Fig. 11. Green fluorescent protein-modified phospholipid synthesized via native chemical ligation with a peptide-modified phospholipid. the phospholipid. Using this technique, e.g. the fusion of an influenza virus with a model membrane was studied and it was found that hemagglutinin, the fusion protein, is transiently connected to both the virus envelope and the target membrane but it will only penetrate halfway through the latter.[11]

Recently, the same protein was studied by the group of Furuta and Kan. They reported on a family of nonnatural phospholipids bearing different headgroups attached via a convenient oxime-linker (Fig. 9). ${ }^{[12]}$ Groups such as pyrene and $\mathrm{Cu}(\mathrm{II})-$ chelates were used to cleave the membrane protein hemagglutinin. The cleavage only occured when both the nonnatural phospholipid and the hemagglutinin were present in the same vesicle. In a control experiment, the free headgroups themselves were added externally without showing any effect. ${ }^{[12]}$

\section{Biological Studies}

The frontier between pure biophysical to biological studies is easily crossed, especially when working with phospholipids. Currently, the field of solid-supported membranes as models for the cell surface is receiving a great deal of attention. ${ }^{[13]} \mathrm{Mi}$ cropatterning techniques offer a wide open field of flat, fixed geometries. ${ }^{[14]}$ In particular, controlled photocrosslinking of diyne phospholipids shows great promise (see Fig. 3). Illumination of the sample through a mask can provide regions containing unpolymerized phospholipids in a surface of polymerized phospholipids. The unpolymerized phospholipids can be washed away and the holes be refilled with natural phospholipids creating constraint regions of free floating bilayer membranes. ${ }^{[15]}$ As proteins can be reconstituted in similar supported membranes, ${ }^{[16]}$ a new, attractive array tool for biological and medicinal assays will become available (Fig. 10).

Fluorescence microscopy remains a key technology in studying membranes but besides the commercially available phospholipid probes only few new fluorescent phospholipid probes have appeared in the literature. The groups of Bertozzi and Groves recently used native chemical ligation to couple green fluorescent protein to a phosphatidyl ethanolamine and thus created a mimic of a glycosylphosphatidylinositol (GPI)-linked protein (Fig. 11). ${ }^{[17]}$ The molecule stably incorporated into a supported bilayer membrane and the lateral mobility of the probe could be monitored.

In conclusion, the study of nonnatural phospholipids remains an attractive field of research in a highly interdisciplinary environment. Each step brings us closer to a deeper understanding of our plasma membranes, knowledge that is as important to basic science as it is to applied medicine.

\section{Acknowledgments}

The author would like to thank the editorial board of CHIMIA for the opportunity to publish in this issue.

Received: January 12, 2009
[1] M. R. Wenk, Nat. Rev. Drug Discov. 2005, 4, 594.

[2] P. P. M. Bonsen, G. J. Burbach-Westerhuis, G H. de Haas, L. L. M. van Deenen, Chem. Phys. Lipids 1972, 8, 199.

[3] a) D. Chapman, Langmuir 1993, 9, 39; b) A Singh, J. M. Schnur, Polymer. Adv. Technol. 1994, 5,358

[4] a) H.-H. Hub, B. Hupfer, H. Koch, H. Ringsdorf, Angew. Chem. 1980, 92, 962; b) D. S. Johnston, S. Sanghera, M. Pons, D. Chapman, Biochim. Biophys. Acta 1980, 602, 57.

[5] H. Ringsdorf, B. Schlarb, J. Venzmer, Angew. Chem. Int. Ed. Engl. 1988, 27, 113.

[6] K. Ishihara, T. Ueda, N. Nakabayashi, Polym. J. 1990, 22,355

[7] Z. Huang, F. C. Szoka, Jr., J. Am. Chem. Soc. 2008, 130, 15702

[8] S. L. Regen, Curr. Opin. Chem. Biol. 2002, 6, 729.

[9] C. M. Gupta, C. E. Costello, H. G. Khorana, Proc. Natl. Acad. Sci. USA 1979, 76, 3139.

[10] A. Seelig, J. Seelig in 'Encyclopedia of Physical Science and Technology', Ed. R. A. Meyers, Academic Press, New York, 2001, p. 355

[11] J. Brunner, Annu. Rev. Biochem. 1993, 62, 483.

[12] T. Furuta, M. Mochizuki, M. Ito, T. Takahashi, T. Suzuki, T. Kan, Org. Lett. 2008, 10, 4847.

[13] M. Tanaka, E. Sackmann, Nature 2005, 437, 656.

[14] J. T. Groves, S. G. Boxer, Acc. Chem. Res. 2002 $35,149$.

[15] T. Okazaki, T. Inaba, Y. Tatsu, R. Tero, T. Urisu, K. Morigaki, Langmuir 2009, 25, 345.

[16] B. A. Cornell, V. L. B. Braach-Maksvytis, L. G. King, P. D. J. Osman, B. Raguse, L. Wieczorek, R. J. Pace, Nature 1997, 387, 580.

[17] M. J. Grogan, Y. Kaizuka, R. M. Conrad, J. T. Groves, C. R. Bertozzi, J. Am. Chem. Soc. 2005, 127, 14383. 